\title{
PLANE CURVES WHOSE SINGULAR POINTS ARE CUSPS
}

\author{
HISAO YOSHIHARA
}

(Communicated by Louis J. Ratliff, Jr.)

\begin{abstract}
Let $C$ be an irreducible curve of degree $d$ in the complex projective plane. We assume that each singular point is a one place point with multiplicity 2 or 3 . Let $\sigma$ be the sum of "the Milnor numbers" of the singularities. Then we shall show that $7 \sigma<6 d^{2}-9 d$. This gives a necessary condition for the existence of such a curve, for example, if $C$ is rational, then $d \leq 10$.
\end{abstract}

1. Introduction. Let $C$ be an irreducible curve of degree $d$ in the complex projective plane $\mathbf{P}^{2}$. We assume that $C$ is not smooth and each singular point is a cusp (i.e., one place point) with multiplicity 2 or 3 . Let $P_{i}$ be the singular point and $\mu_{i}$ be the Milnor number of the singularity at $P_{i}$, where $i=1,2, \ldots$ Then, putting $\sigma=\sum_{i} 6\left[\mu_{i} / 6\right]$, where [] denotes the Gauss' symbol, we have the following inequality (cf. $[\mathbf{1}, \S 5])$.

THEOREM 1. Let $C$ be the above-mentioned curve. Then $7 \sigma<6 d^{2}-9 d$.

There has been a problem whether there exist curves in $\mathbf{P}^{2}$ with assigned numerical characters satisfying the genus formula of Clebsch $[\mathbf{3}, \S 9.1]$. More than half a century ago Lefschetz and Zariski studied such a problem for Plückerian characters $[4,9]$. Now, let $g$ be the genus of the normalization of $C$. Then from the above theorem we obtain the following inequality.

COROLlaRY 2. $14 g \geq d^{2}-12 d+16$. Especially, if $C$ is rational, then $3 \leq d \leq$ 10.

REMARK 3. The genus formula implies $\lim \sup _{d \rightarrow \infty} \sigma / d^{2} \leq 1$, but the above theorem gives a better inequality $\lim \sup _{d \rightarrow \infty} \sigma / d^{2} \leq 6 / 7$ (cf. $[\overline{\mathbf{2}}, \S 8]$ ).

2. Miyaoka's inequality. The key to the proof of the theorem is a result of Miyaoka [5, Corollary 1.2]. Before stating it we list the necessary notations.

$X$ : a projective nonsingular surface,

$D$ : a reduced divisor on $X$ with normal crossings,

$K$ : the canonical divisor on $X$,

$e(V)$ : the topological Euler characteristic of a variety $V$. Then the result is stated as follows.

LEMMA 1. If $K+D$ is numerically equivalent to an effective rational divisor, then $3\{e(X)-e(D)\} \geq(K+D)^{2}$. If the equality holds, then $D$ is a semistable curve.

Received by the editors May 11, 1987 and, in revised form, June 6, 1987.

1980 Mathematics Subject Classification (1985 Revision). Primary 14H45; Secondary $14 \mathrm{H} 20$.

Key words and phrases. Plane curve, cusp, Milnor number. 
The outline of the proof of Theorem 1 is as follows. We consider the composition of $k$ blow-ups $f: X \rightarrow \mathbf{P}^{2}$ satisfying that $k$ is minimal in order that the divisor $D$ has normal crossings, where $D$ is the reduced divisor obtained from $f^{*}(C)$. Then we note that $K+D$ is numerically equivalent to an effective rational divisor if and only if the logarithmic Kodaira dimension $\bar{\kappa}\left(\mathbf{P}^{2}-C\right) \geq 0$ [3, §11.2]. Making use of the above result when $\bar{\kappa}\left(\mathbf{P}^{2}-C\right) \geq 0$, we shall prove Proposition 4 below, from which Theorem 1 will be easily deduced.

3. Proofs. We denote by $2(m), 3(n)$ and $3(n)+2$ the sequences $(2, \ldots, 2)$, $(3, \ldots, 3)$ and $(3, \ldots, 3,2)$ respectively, where 2 appears $m$ times in the first sequence and 3 appears $n$ times in the latter two sequences. For a cusp $P$ on $C$ the sequence of the multiplicities of all the infinitely near singular points of $P$ will be called the sequence of $P$ for short. Let $P_{i}$ be the cusp on $C$ with multiplicity 2, where $i=1, \ldots, r$, and let $2\left(m_{i}\right)$ be the sequence of it. Then the singularity at $P_{i}$ is analytically equivalent to the one at $(0,0)$ defined by $y^{2}+x^{2 m_{i}+1}=0$. Hence the Milnor number of the singularity at $P_{i}$ is $2 m_{i}$. On the other hand, let $Q$ be the cusp with multiplicity 3 . Then there are two cases, i.e., the sequence of $Q$ is $3(n)$ or $3(n)+2$ for some $n \geq 1$. Let $Q_{i}$ be the cusp with the sequence $3\left(n_{i}\right)$ [resp. $3\left(n_{i}\right)+2$ ], where $i=1, \ldots, s$ [resp. $\left.i=s+1, \ldots, s+t\right]$.

LEMMA 2. The singularity at $Q_{i}$ is equivalent to the one at $(0,0)$ defined by $y^{3}+a(x) y+x^{N_{i}}=0$, where $N_{i}=3 n_{i}+1$ for $i=1, \ldots, s\left[\right.$ resp. $N_{i}=3 n_{i}+2$ for $i=s+1, \ldots, s+t]$ and $a(x)$ is convergent power series with the order $\geq 2 n_{i}+1$ [resp. $\left.2 n_{i}+2\right]$.

PrOOF. Applying the Weierstrass preparation theorem and next doing a Tschirnhaus transformation, we can put the local equation of $C$ at $Q_{i}$ into the form $y^{3}+a(x) y+b(x)=0$, where $a(x)$ and $b(x)$ are convergent power series. Since the singularity is cuspidal, by doing blow-ups at the infinitely near singular points of $Q_{i}$, we infer that ord $a(x) \geq 2 n_{i}+1$ [resp. $2 n_{i}+2$ ] and ord $b(x)=3 n_{i}+1$ [resp. $\left.3 n_{i}+2\right]$. Then, by taking new coordinates, we arrive at the conclusion.

We put $m=\sum_{i=1}^{r} m_{i}$ and $n=\sum_{i=1}^{s+t} n_{i}$. From the above lemma the following one is obtained by a simple calculation.

LEMMA 3. The Milnor number of the singularity at $Q_{i}$ is $6 n_{i}$, where $i=1, \ldots, s$ [resp. $6 n_{i}+2$, where $\left.i=s+1, \ldots, s+t\right]$. Hence $\sigma=\sum_{i=1}^{r} 6\left[m_{i} / 3\right]+6 n$.

The "number" of the singular points of $C$ is $m+n+t$. We have the following estimate.

PROPOSITION 4. $2 d^{2}-3 d>5 m+14 n+s+6 t$.

From this proposition we infer readily the theorem, so we shall prove this one hereafter.

Let $f: X \rightarrow \mathbf{P}^{2}$ be the composition of $k$ blow-ups such that $k$ is minimal in order that the divisor $D$ has normal crossings, where $D$ is the reduced divisor obtained from $f^{*}(C)$. Then the dual graphs of (i) $f^{-1}\left(P_{i}\right)$, (ii) $f^{-1}\left(Q_{i}\right)$ for $i=1, \ldots, s$, and (iii) $f^{-1}\left(Q_{i}\right)$ for $i=s+1, \ldots, s+t$, are described as follows respectively, where $\circ$ denotes the curve with the self-intersection number -2 , and the number beside a curve indicates the self-intersection number. 
(i)

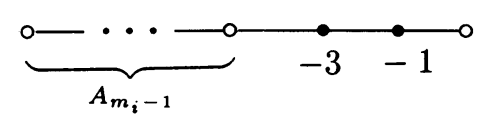

(ii)

(iii)
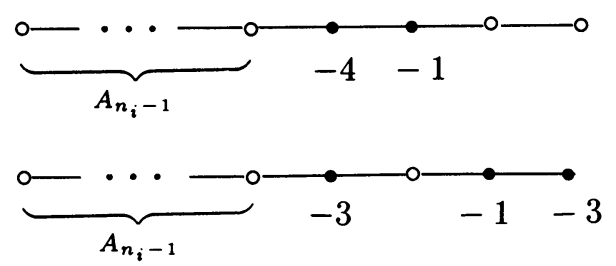

Thus $k=m+n+2 r+3 s+3 t$. The topological Euler characteristic $e(X)=3+k$ and $e(D)=2-2 g+k$. Let $C^{\prime}$ be the proper transform of $C$ by $f^{-1}$, then the self-intersection number $C^{\prime 2}$ is $d^{2}-(4 m+2 r+9 n+3 s+6 t)$. Let $K$ be the canonical divisor on $X$, then $C^{2}+K C^{\prime}=2 g-2$ and $K^{2}=9-k$. Since $D(K+D)=2 g-2$ and $K D=s+t=K C^{\prime}$, we have that $(K+D)^{2}=7+s+t+K C^{\prime}+2 g-k$. Hence the following relations hold true.

LEMMA 5. $e(X)=3+k, e(D)=2-2 g+k$ and $(K+D)^{2}=4 g+5+s+t-k-C^{2}$.

We shall prove the proposition by examining the following cases separately:

(1) $r+s+t \geq 2$ or $g \geq 1$,

(2) $r+s+t=1$ and $g=0$. First we treat case (1). Thanks to [6], if $C$ is the curve with the property $(1)$, then $\bar{\kappa}\left(\mathbf{P}^{2}-C\right) \geq 0$. Hence, applying Lemma 1 and noting that $D$ is not a semistable curve, we infer from the above results that $d^{2}+2 g>$ $3 m+8 n+s+4 t+2$. Using the genus formula $2 g=(d-1)(d-2)-2 m-6 n-2 t$, we arrive at the inequality of Proposition 4.

Next we treat the case (2). Let $P$ be the unique cusp and put $e=\operatorname{mult}_{P} C$. In case $d \geq 3 e$, then $\bar{\kappa}\left(\mathbf{P}^{2}-C\right)=2[\mathbf{7}$, Proposition 1$]$. So that the proof is the same as in the case (1). On the contrary, in case $d<3 e$, then the validity of the inequality is checked directly by using the genus formula. Thus the proof of Proposition 4 is complete.

Putting the proposition and the genus formula together, we get the following inequality.

PROPOSITION 6. $14 g>d^{2}-12 d+14+m+3 s+4 t$.

Then Corollary 2 is clear.

4. Relevant results. In case $d$ is a multiple of 3 , i.e., $d=3 h$, we consider the minimal resolution $S$ of the triple covering of $\mathbf{P}^{2}$ branched along $C$. Observing the Picard number of $S$, we can show the following.

REMARK $7.5 \sigma \leq 39 h^{2}-27 h+6$ and $10 g \geq 6 h^{2}-18 h+4$.

Note that, if $h \leq 17$, then the former inequality is better than the one in Theorem 1.

Now here is a conjecture.

ConjeCture 8. If $C-\{P\} \cong \mathbf{A}^{1}$, then $d<3 e$, where $e=\operatorname{mult}_{P} C$.

In case $e=2$, this conjecture holds true [8]. If $e=3$, then $d \leq 10$ by Proposition 6. Moreover by Remark 7 we see that $d \neq 9$. So it remains to be proved that $d \neq 10$. 
Finally we present an example, which is proved by simple but laborious computations.

EXAMPLE 9. Let $C$ be as in the above conjecture. Suppose that $d=6$ and $e=3$. Then the sequence of $P$ is $3(3)+2$ and $\sigma=18$. The curve $C$ is projectively equivalent to $C_{t}$ for some $t \in \mathbf{C}$, which is defined by

$$
\left(y-x^{2}\right)^{3}+t\left(y-x^{2}\right) y^{4}+x y^{5}=0 .
$$

Two curves $C_{t}$ and $C_{s}$ are projectively equivalent if and only if $t^{5}=s^{5}$.

\section{REFERENCES}

1. F. Hirzebruch, Some examples of algebraic surfaces, Contemp. Math., vol. 9, Amer. Math. Soc., Providence, R. I., 1982, pp. 55-71.

2. _ـ Singularities of algebraic surfaces and characteristic numbers, Contemp. Math., vol. 58, Amer. Math. Soc., Providence, R. I., 1986, pp. 141-155.

3. S. Iitaka, Algebraic geometry, an introduction to birational geometry of algebraic varieties, Graduate Texts in Math., no. 76, Springer-Verlag, Berlin and New York, 1981.

4. S. Lefschetz, On the existence of loci with given singularities, Trans. Amer. Math. Soc. 14 (1913), 23-41.

5. Y. Miyaoka, The maximal number of quotient singularities on surfaces with given numerical invariants, Math. Ann. 268 (1984), 159-171.

6. I. Wakabayashi, On the logarithmic Kodaira dimension of the complement of a curve in $\mathbf{P}^{2}$, Proc. Japan Acad. 54A (1978), 157-162.

7. H. Yoshihara, On plane rational curves, Proc. Japan Acad. 55A(1979), 152-155.

8.

9. O. Zariski, On the non-existence of curve of order 8 with 16 cusps, Amer. J. Math. 53 (1931), 309-318.

Faculty of General Education, Nilgata University, Nigata, 950-21 Japan 